\title{
Public Health Measures (Lockdown) and its Psychological Impact: A General Population Study of Mental Health Difficulties during the Covid-19 Pandemic and Resultant Lock Down
}

\author{
Jessy Fenn ${ }^{1}$, Noble Chacko ${ }^{1}$, Tony Thomas ${ }^{1}$, Varghese K Varghese ${ }^{2}$, Sanju George ${ }^{3}$ \\ ${ }^{1}$ Assistant Professor, ${ }^{2}$ Dean, Department of Psychology, ${ }^{3}$ Professor of Psychiatry and Psychology and Consultant Psychiatrist, \\ Rajagiri Centre of Behavioural Science and Research, \\ Rajagiri College of Social Sciences (Autonomous), Kalamassery Kochi, Kerala, India \\ E-mail: sanjugeorge531@gmail.com
}

\begin{abstract}
Public health measures taken to prevent the spread of pandemics such as COVID-19 can negatively impact on the mental health of individuals. This study reports perceived stress, sources of stress, psychological distress, and posttraumatic stress disorder in $\mathbf{1 0 7 3}$ adults from Kerala, India during the lockdown phase. Methods: Data was gathered using these questionnaires: Perceived Stress Scale, Kessler Psychological Distress Scale and PC-PTSD scale. Results: Descriptive analyses revealed that $65.7 \%$ of participants experienced increased stress levels due to the lockdown; with $57.4 \%$ experiencing moderate and $8.3 \%$ of experiencing severe stress. Discussion: The major sources of stress were anxiety about the future, finance-related, frustration of movement due to restriction and anxiety about COVID. Although there were no cases of PTSD, about $30 \%$ of the sample suffered from severe psychological distress. The above issues should be addressed by policy makers and health care professionals in trying to ameliorate the psychological distress experienced by people during pandemic-related lockdown in the future.

Keywords: Coping strategies; COVID-19; Perceived stress; Psychological distress; PTSD; Stress.
\end{abstract}

\section{INTRODUCTION}

India has had 217,000 confirmed cases of COVID-19 and 6.075 deaths (as of $5^{\text {th }}$ June 2020) from its 29 States and 7 Union Territories. ${ }^{[1]}$ A key public health measure announced by the Government of India to counter the spread of the COVID-19 pandemic was a country-wide lockdown from $24^{\text {th }}$ of March 2020, until $17^{\text {th }}$ of May 2020. This involved limiting the number of people getting out of their homes, shutting down of shops and, preventing people from all religious and social gatherings, restricting access to public transport and so on. These restrictions were designed to ensure the maintenance of best possible physical distance between people and thereby reduce transmission of the virus. As these public health measures had to be implemented hastily, they resulted in many unavoidable deficiencies including its negative impact on the psychological well-being of individuals. We carried out a study to ascertain the mental health status (focussing on stress, depression, anxiety, and PTSD) of people during the lockdown in Kerala. Kerala is the south-western most state of India with a population of approximately 35 million people and Human Development Index of 0.78 (the highest in India). Perhaps, Kerala's experience of recent epidemics such as Nipah meant that Kerala was better prepared to handle COVID-19 than the rest of India. ${ }^{[2]}$

\section{METHODS}

Data was drawn through standardised instruments to assess perceived stress, depression, anxiety and PTSD using Perceived Stress Scale (PSS-4), ${ }^{[3]}$ Kessler Psychological Distress Scale (K6), ${ }^{[4]}$ preferred for screening mood or anxiety disorders and PC- PTSD-4. ${ }^{[5]}$ Apart from the PSS4 , a self - report questionnaire captured stress levels before and after the lockdown on a 10 - point Scale. Sources of stress and coping strategies based on the COPE inventory ${ }^{[6]}$ were also captured by listing potential stressors and coping strategies relevant to this lockdown period created by the researchers. Apart from English, the survey was also translated using forward- backward translation to Malayalam, the local language. The survey tool was created in Google forms and was distributed primarily through social media such as What's App to people across Kerala. The survey was open from $15^{\text {th }}$ to20th of April 2020, as lockdown restrictions were being eased in a few districts of Kerala from the 21st April. We collected 1073 responses, and these were analysed. Data analysis was performed using SPSS version 20 importing the MS Excel sheet from the Google forms. Correlational analysis was used to assess the association between perceived stress and the other variables such as coping strategies and depression, and the student's t test for independent samples and one-way ANOVA were used to compare the mean values of stress and psychological distress with the various demographic variables. Of the total of 1073 respondents, 59\% were women and 41\%were men; and 55\% were aged between 17 and 25 years, with the remainder somewhat equally distributed among other age groups.

\section{RESULTS}

Stress experienced by the respondents was measured using the Perceived Stress Scale (PSS-4); the details of their demographic attributes are presented in Table I. 
TABLE I SHOWING THE NUMBER (N) AND PROPORTION OF PARTICIPANTS IN PERCENTAGE (\%), BELONGING TO EACH LEVEL OF STRESS

\begin{tabular}{|c|c|c|c|c|c|}
\hline \multicolumn{6}{|c|}{ Level of Stress based on Perceived Stress Scale 4 (PSS 4) } \\
\hline & & Low & Mild & Moderate & Severe \\
\hline Number & \multirow{4}{*}{$(\%)$} & 77.(7.2) & $365(34.3)$ & $61657.4)$ & 89(8.3) \\
\hline Mean PSS & & 2.96 & 3.24 & 7.72 & 12.19 \\
\hline \multirow{2}{*}{ SD } & & 1.41 & 1.68 & 1.28 & 1.50 \\
\hline & & $\mathrm{N}(\%)$ & $\mathrm{N}(\%)$ & $\mathrm{N}(\%)$ & $\mathrm{N}(\%)$ \\
\hline \multirow{2}{*}{ Gender } & Males & $29(6.2)$ & $226(51.8)$ & $164(37.6)$ & $19(4.4)$ \\
\hline & Females & $48(7.7)$ & 338 (53.9) & $226(36)$ & $15(2.4)$ \\
\hline \multirow{5}{*}{ Age group } & $18-25$ & $59(9.8)$ & 370 (61.3) & 169 (28) & $6(1)$ \\
\hline & $26-35$ & $8(4.8)$ & $101(60.1)$ & $56(33.3)$ & $3(1.8)$ \\
\hline & $36-45$ & $2(1.9)$ & 31 (29) & $70(65.4)$ & $4(3.7)$ \\
\hline & $46-55$ & $4(4.3)$ & 37 (39.8) & $47(50.5)$ & $5(5.4)$ \\
\hline & Above 55 & $4(4.1)$ & $29(29.6)$ & $49(50)$ & $16(16.3)$ \\
\hline \multirow[t]{4}{*}{ Religion } & Hindu & $36(9.5)$ & $204(54.1)$ & $126(33.4)$ & $11(2.9)$ \\
\hline & Christian & $31(5.8)$ & $281(52.7)$ & $206(38.6)$ & $15(2.8)$ \\
\hline & Muslim & 9 & 67 (54.9) & $39(32)$ & $7(5.7)$ \\
\hline & No religion & 1 & $16(42.1)$ & $20(52.6)$ & $1(2.6)$ \\
\hline \multirow{3}{*}{ Residence } & City / Urban & $39(7.1)$ & $280(51.3)$ & $209(38.3)$ & $18(3.3)$ \\
\hline & Town / Semi-urban & $18(6.6)$ & $149(55)$ & $97(35.8)$ & $7(2.6)$ \\
\hline & Village / Rural & $20(7.9)$ & $139(54.9)$ & 85 (33.6) & $9(3.6)$ \\
\hline
\end{tabular}

Scores of the PSS-4 between 0-5 were coded as mild, 6-10 as moderate and 11-16 as high as instructed in the scale manual. The mean value of PSS-4 for the sample was 7.7. PSS-4 scale does not provide norms but previous studies from several countries have shown average mean values of populations to be between 5 and 6.5 (Vallejo et al, 2018). This indicates that there was a moderate level of stress experienced by the sample. Of the respondents, $8.3 \%$ had experienced high stress, $57.4 \%$ experienced moderate stress and $34.3 \%$ experienced only mild stress. There was no significant difference in the stress level experienced between males (Mean=6.39, $\mathrm{SD}=3.20$ and females ( Mean=6.67, $\mathrm{SD}=2.91$ ) as seen from an independent sample $\mathrm{t}$-test $(\mathrm{t}=-1.4$, sig=0.16,). Stress was significantly negatively related to age as seen from pearson's correlation coefficient of -0.18 and significant at 0.01 level; the more the age, the less the stress. The mean score of the stress rating before lockdown was 4.12, and 21 days into lockdown the stress rating was 4.56 . While these stress levels could be seen as moderate, the difference between the before during rating was found to be statistically significant through a paired $t$ test $(\mathrm{t}=4.63, \mathrm{p}<.001)$. This indicates there was an increase of stress after lockdown started.

The difference between every respondent's stress before and during lockdown revealed that while $51.5 \%$ of respondents showed an increase in stress during lockdown, for $31.5 \%$ there was also a marked reduction in stress. Free from the hassles of work, travel and other demands of daily life, the lockdown was perceived as less stressful. For about $17.4 \%$ of the sample, stress before and during lockdown was the same. The primary sources of stress were found to be anxiety about what was happening around the world (53\% of respondents) followed by the worry about the future in terms of what would happen after the lockdown is over (48\%). This was followed by worries about future of job/ business (43\%), financial crunch (41\%) and worries closer to home. Since only 3 deaths had occurred in Kerala, fears of contracting COVID were not as high and it came only in 6th place. Loneliness, which is one of the main worries in many countries was not as severe in India where people lived with families and close to others. It was, however, a problem for $26 \%$ of the people, and mainly men. One of the outcomes of stress is anxiety and depression, which was measured by Kessler Distress Scale, K6. The mean of the Kessler K-6 score for the sample was 8.05, indicating moderate depression. Severe mental illness, defined as K6 scores $=/>13$, is estimated to afflict about $6 \%$ of adults (Tomitaka et al, 2019). The mean and the spread of the K-6 distress scale indicated that $22 \%$ were struggling with mental distress during the lock down period (Table 2). Women $(\mathrm{M}=8.6)$ had significantly $(\mathrm{t}=-3.5, \mathrm{p}<0.000)$ higher distress than men $(M=7.2)$ and unmarried people $(M=9.1)$ significantly $(\mathrm{t}=7.52, \mathrm{p}<0.000)$ higher than married people $(\mathrm{M}=6.2)$. There was a highly significant negative correlation of -0.28 between age and K6 scores. 
TABLE II DISTRIBUTION OF K6 SCORES IN THE SAMPLE

\begin{tabular}{|l|c|c|}
\hline \multicolumn{3}{|c|}{ K-6 Kessler Distress Scale } \\
\hline Level (score) & $\mathrm{N}$ & $\%$ \\
\hline Very High- above 18 & 69 & $6 \%$ \\
\hline High Depression (13-18) & 173 & $16 \%$ \\
\hline Moderate depression (6-12) & 234 & $22 \%$ \\
\hline No depression (0-6) & 419 & $39 \%$ \\
\hline
\end{tabular}

PC-PTSD scores indicated that there was no incidence of PTSD in the sample which was partly understandable as there were only 497 cases of COVID in the state.

\section{DISCUSSION}

Various coping strategies were adopted by the participants to reduce, minimize, master, or tolerate the stress of the lockdown period. The common coping strategies were ensuring COVID prevention hygiene measures and social distancing (75\%), communication with family (56\%), watching movies/reading (54\%), being updated on COVID news (52\%), social media jokes and conversations (50\%), and stocking on food (49\%).Although the top five coping strategies used by men and women were the same, women were significantly higher $(\mathrm{p}<0.05)$ in the intensity of the coping strategy adopted for 13 of the strategies; which in the order of difference in magnitude were: praying, stocking food, sleep, watching movies, listening to religious talks, hygiene practices, exercises/walks, indulging in hobbies, self-discovery exercises, reading about COVID prevention, and communicating with family. Also, a more careful look at the type of strategies adopted overall revealed more problem-focused strategies such as active coping, planning, as well as emotional support to have been more frequently adopted. Coping strategies such as substance abuse and venting were less used. TV news was the primary source of information about COVID for $86 \%$ of respondents, followed by $62 \%$ from newspapers, $44 \%$ from online searches, $43 \%$ from what's app messages and $42 \%$ from friends and family.

These findings of increased stress and anxiety during the lockdown phase agree with previous studies that suggested higher general psychological symptoms, emotional disturbance, and exacerbation in pre-existing mental health difficulties ${ }^{[7,8]}$ stress during times of pandemic - related quarantine. A unique strength of our study is that this is the first of its nature from India to look at stress, coping, coping strategies, and depression among the general population during the COVID-19 pandemic and its lockdown. Further, a sample size of over 1000 gives valid and generalizable findings. However, as this study was limited to Kerala, it could be argued that these findings may not be generalizable to other parts of India. Nevertheless, having seen the sociodemographic spread of our sample, we make the case that these findings are representative of the wider population of India and will offer useful pointers to public health policy makers.

\section{CONCLUSION}

In summary, health care providers and public health policy makers need to acknowledge that pandemics (when associated with lock down) negatively impact on the psychological well-being of individuals. In the event of similar future pandemics, strategies need to be in place to safeguard the psychological well-being of individuals by offering them timely and appropriate psychological support, as well as taking the appropriate steps in the effective management of those already affected psychologically.

\section{REFERENCES}

[1] COVID-19 cases in India. Ministry of Health and Family Welfare, India. Available from: https://www.mohfw.gov.in/. Last accessed on June 52020.

[2] Rahim, AA, Chacko, TV. (2019). Nipah outbreak in North Kerala What worked? Insights for future response and recovery based on examination of various existing frameworks. Indian Journal of Public Health, 63 (3), 261 - 264.

[3] Cohen, S, Kamarck, T, Mermelstein, R.A global measure of perceived stress. (1983). Journal of Health and Social Behaviour, 24, 385-396.

[4] Kessler, RC, Andrews, G, Colpe, LJ, Hiripi, Mroczek, EDK, Normand, SLT, Walkters, EE, Zaslavsky, AM. (2020). Short Screening Scales to Monitor Population Prevalences and Trends in Non-Specific Psychological Distress. Cambridge University Press.

[5] Prins A, Bovin MJ, Kimerling R, Kaloupek DG, Marx BP, Pless Kaiser A, Schnurr PP. (2015). Primary Care PTSD Screen for DSM-5 (PC-PTSD-5) [Measurement instrument]. Available from https://www.ptsd.va.gov.

[6] Carver, CS, Scheier, MF, Weintraub, JK. (1989). Assessing coping strategies: a theoretically based approach. Journal of Personality and Social Psychology, 56, 267-283.

[7] Yoon, MK, Kim, SY, Ko, HS, Lee, MS. (2016). System effectiveness of detection, brief intervention and refer to treatment for the people with post-traumatic emotional distress by MERS: a case report of community-based proactive intervention in South Korea. International Journal of Mental Health Systems, 10- 51.

[8] Stress and Coping. Centres for Disease Control and Prevention. (2020). Available from: https://www.cdc.gov/coronavirus/2019ncov/daily-life-coping/managing-stress-anxiety.html.Last accessed on 30 April 2020. 